\title{
DUAL IMMUNOHISTOCHEMISTRY WITH CD34 WILL ENHANCE THE C4d SENSITIVITY AND SPECIFICITY ON RENAL ALLOGRAFT BIOPSY
}

\author{
Ashokkumar Raghupathy', Arunkumar K², S. P. Arun Kumar ${ }^{3}$ \\ ${ }^{1}$ Assistant Professor, Department of Pathology, Sri Venkateshwaraa Medical College Hospital and Research Centre, Ariyur. \\ ${ }^{2}$ Consultant Urologist, RG Urology Hospitals, Chennai. \\ 3Professor, Department of Pathology, Sri Venkateshwaraa Medical College Hospital and Research Centre, Ariyur.
}

ABSTRACT
BACKGROUND
Renal transplantation is one of the lifesaving modality and the survival of the graft is very important. Graft rejection in the form of
antibody mediated or humoral rejection (AMR) is getting more importance as unlike acute cellular rejection (ACR) it has few
diagnostic morphological features in the biopsy and these cases are resistant to anti-rejection therapy with a worse prognosis.

\section{MATERIALS AND METHODS}

A consecutive as well as retrospective study conducted in the department of Pathology, Sri Venkateshwaraa Medical College Hospital and Research Centre, Puducherry on the allograft biopsies received between January 2014 and December 2015 were included randomly and analysed in respect to clinical, histological and the immunohistochemical detection of C4D deposition in the Peritubular Capillaries (PTC) on Renal Allograft rejection biopsies. Immunostaining was done by double labelling method $^{1}$ of avidinbiotin technique using monoclonal antibodies to C4d and CD34.

\section{RESULTS}

Double immunolabeling was mainly used to delineate the peritubular capillaries (By CD34), which is usually very difficult to locate on routine immunohistochemistry. It also helps to define the $\mathrm{C} 4 \mathrm{~d}$ positive cases and to differentiate between the focal and diffuse C4d positive cases. We compared the degree of $\mathrm{C} 4 \mathrm{~d}$ deposition with different grades of acute and chronic rejection according to Banff $2003^{2}$ criteria. We also analysed the prognostic significance of C4d deposition on peritubular capillaries by univariate analysis with the response to anti-rejection treatment.

\section{CONCLUSION}

Dual IHC will reduce the chances of false negative detection of humoral components in allograft biopsy.

\section{KEYWORDS}

Banff, IHC, Acute/Chronic, Humoral Rejection, C4d, CD34.

HOW TO CITE THIS ARTICLE: Raghupathy A, Arunkumar K, Kumar SPA. Dual immunohistochemistry with CD34 will enhance the C4d sensitivity and specificity on renal allograft biopsy. J. Evolution Med. Dent. Sci. 2016;5(88):6541-6545, D0I: $10.14260 /$ jemds/2016/1480

\section{BACKGROUND}

More than 30000 renal transplants are performed annually worldwide. $^{3}$ Diabetes, systemic hypertensive vascular diseases and the various glomerular diseases, most commonly IgA nephropathy ${ }^{4}$ are the commonly listed causes for renal failure. Prevention of chronic rejection still remains a major hurdle in improving long-term allograft survival. The introduction of the potent immunosuppressive drug cyclosporine in the late 1970's increased the rates of success in renal transplants. Innovative therapies, such as antithymocyte globulin (ATG), anti-CD3 monoclonal antibody (OKT3) and mycophenolate, tacrolimus have markedly increased graft survival.

Incidence of clinically evident acute rejection are $11 \%$ to $16 \%$ of graft loss in the first year and it affects $12 \%$ to $18 \%$ of recipients of live related donors, $15 \%$ to $25 \%$ of deceased donors.5,6 In view of these observations, Banff (1997)

Financial or Other, Competing Interest: None.

Submission 28-09-2016, Peer Review 22-10-2016,

Acceptance 28-10-2016, Published 02-11-2016.

Corresponding Author:

Dr. Ashokkumar Raghupathy,

No. 13, Muslim Street, Murugan Arul Hospital

Thiruttani-631209.

E-mail: ashokspp@gmail.com

DOI: $10.14260 /$ jemds $/ 2016 / 1480$

(c) $($ ) $\$$ classification was revised in $2003^{2}$ and 2005 incorporating morphological criteria, supported by immunopathological details for diagnosing humoral rejection.

\section{Morphologically the changes are classified ${ }^{2}$ into \\ - Borderline. \\ - Humoral or antibody mediated/cellular rejection. \\ - Chronic/sclerosing allograft nephropathy.}

Acute humoral reactions are identified on light microscopy and they presented as one of the following three types 2,7 ; acute tubular injury, dilatation of peritubular capillaries with neutrophil margination and the arterial necrosis. Coexistence of both the reaction patterns and the humoral rejection with only tubular injury $(10 \%)$ is also possible. Humoral rejection compared with cellular part reported as worse survival rate ${ }^{8}$; $27 \%$ to $40 \%$ of one-year graft loss (humoral) compared with $3 \%$ to $7 \%$ of one-year graft loss (Cellular). Feucht et al first described about the complement, C4d deposition on renal blood vessels and that could be the evidence as humoral rejection on allograft biopsies.9,10 Peritubular Capillaries (PTC) mainly serves as major site for detectable immunecomplex deposition ${ }^{11,12}$ with complement activation because compared with glomeruli they have less complement protective pathways. C4d deposition in peritubular capillaries is not detected in other immune mediated, inflammatory 
disorders. So it is considered as a 'transplant specific' site for C4d deposition. ${ }^{11,12}$

The degree of C4d deposition on Peritubular Capillaries (PTC) and its correlation with the severity of acute and chronic rejection as well as the response to therapy is controversial.

\section{MATERIALS AND METHODS}

A consecutive as well as retrospective study conducted in the Department of Pathology, Sri Venkateshwaraa Hospitals and Research Institute (Puducherry) on the allograft biopsies received between January 2014 and December 2015 were included. Cases were collected from the RG Urology Hospital, Chennai and followup done with adequate investigations. All the biopsies were indicated and performed for the diagnosis of graft dysfunction. Cases were selected randomly with adequate tissue for both routine histology as well as IHC. Clinical details of primary disease, duration of transplant and the laboratory data were collected from the concerned case files.

Histology was evaluated by using H\&E, PAS (Periodic AcidSchiff) and silver methenamine stains. Morphological diagnosis and the grading of rejection is done by using Banff 2003 criteria $^{2,13,14}$ (Figure 1 to 6). Immunostaining for C4d and CD34 was done by labelled Streptavidin-biotin immunoperoxidase technique ${ }^{1}$ (LSAB) using monoclonal antibody to C4d and CD34 on serial step sections. ${ }^{1}$

Sections are overlaid with adequate amount of diluted (1:100 dilution) primary antibody C4d: Rabbit anti-human C4d, polyclonal IgG obtained from AbD Serotec; 0300-0230. For immunostaining with the second monoclonal antibody CD34, antigen retrieval and serum blocking steps were omitted. Sections were then overlaid with adequate amount of diluted (1:500 dilution) CD34 antibody followed by overnight incubation at $4^{\circ} \mathrm{C}$ in a humid chamber. CD34 antibody (QBEND) was obtained from M/s Dakocytomation, Denmark and the dilution used was 1:500.

Peritubular capillaries and the glomerular capillaries were CD34 positive, stained by Vector SG blue. C4d deposited in peritubular capillaries, glomerular capillaries and in mesangium, were stained by DAB. All the inflammatory cells nuclei and tubular epithelium nuclei were counterstained by Methyl Green. Internal glomerular capillary and mesangium C4d deposition (In both rejection and non-rejection cases) were considered as a positive control $9,11,12$ for our study. All non-rejection protocol biopsies and minimal change disease were considered as a negative control.11,12

C4d can be deposited in both peritubular capillaries and glomerular capillaries in different patterns (Figure 1 to 6). All these patterns are considered to be positive. None of them found to show any difference in clinical outcome. They include,

- Diffuse circumlinear positive capillaries.

- Block positive.

- Fine or coarse granular positive.

C4d positive peritubular capillaries were counted under high power $(40 \mathrm{X})$ with the exclusion of glomerular capillaries, venules and larger arteries C4d positivity. C4d negative PTCs were counted to assess the degree of the $\mathrm{C} 4 \mathrm{~d}$ deposition. It was classified into three grades. ${ }^{15,16}$

- Negative.

- Focal - less than $50 \%$ of the peritubular capillaries were positive.
- $\quad$ Diffuse - more than $50 \%$ of the peritubular capillaries were positive.

\section{Data Analysis}

Detection of C4d deposition in the Peritubular Capillaries (PTC) on Renal Allograft Rejection biopsies. Correlation of C4d deposit with response to anti-rejection treatment. Fisher exact ' $\mathrm{t}$ ' test (Parametric test) was done to compare the $\mathrm{C} 4 \mathrm{~d}$ deposition in different grades of rejection. Univariate analysis (Chi square test) was done to see the prognostic significance of C4d positive cases with anti-rejection treatment.

\section{RESULTS}

In this study 43 cases were male, 7 female and their age groups were between 17 and 51 years; 39 patients (78\%) had live related donor, 10 had live unrelated and 1 had cadaver donor transplantation. End stage glomerular disease, diabetic nephropathy ( 55 to $65 \%$ ) were the common indication for the transplantation and the other causes were chronic interstitial nephritis and adult polycystic kidney disease. Graft dysfunction was manifested from the first week of posttransplant till 5 years.

Each case of renal transplant biopsy was then followed up with a regular treatment protocol for a minimum period of 6 months. Graft survival and prognosis of the patient were analysed in this short followup period. We analysed our treatment results as responders or non-responders. This division was based primarily on the serum creatinine level at the end of the followup period.

All the 50 cases were graded according to Banff 20037,13,14 rejection criteria; 38 cases were found to have any one form of rejection, either the acute or chronic with cellular or humoral components; 12 cases (24\%) were found to have no rejection in our study (Figure 1 to 6 ).

On comparing the $C 4 \mathrm{~d}$ positive peritubular capillaries with different grades of Banff ACR grades, it showed highly uneven distribution among the various grades; 8 cases ( 67 percent) of grade II acute cellular rejection were $C 4 \mathrm{~d}$ positive; 2 cases of grade 0 (no rejection) as per the histological criteria were found to have C4d positive (Table 1).

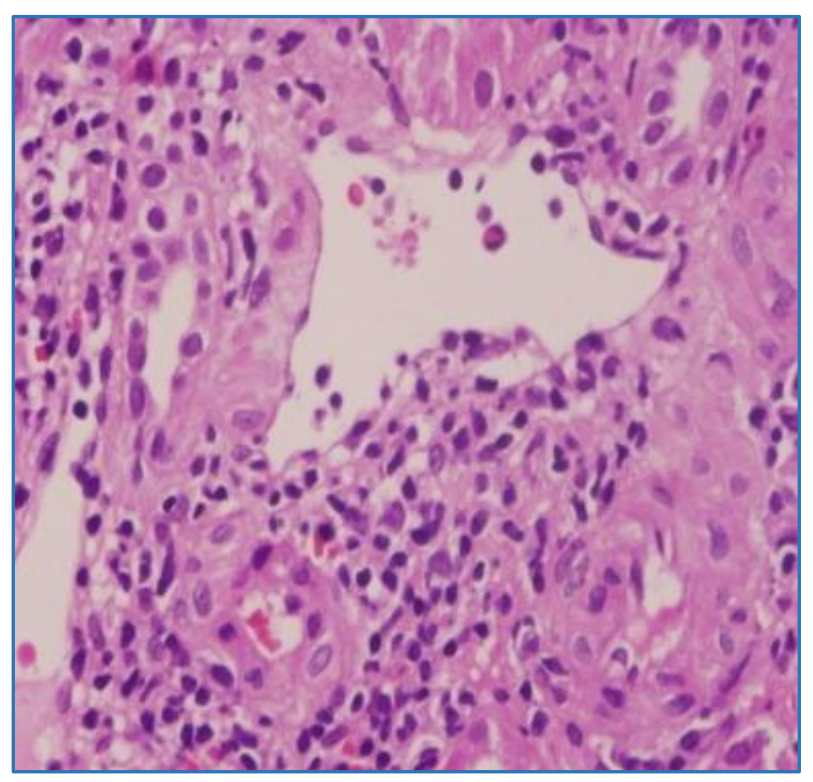

Figure 1. PTC Dilatation and Neutrophil Margination 


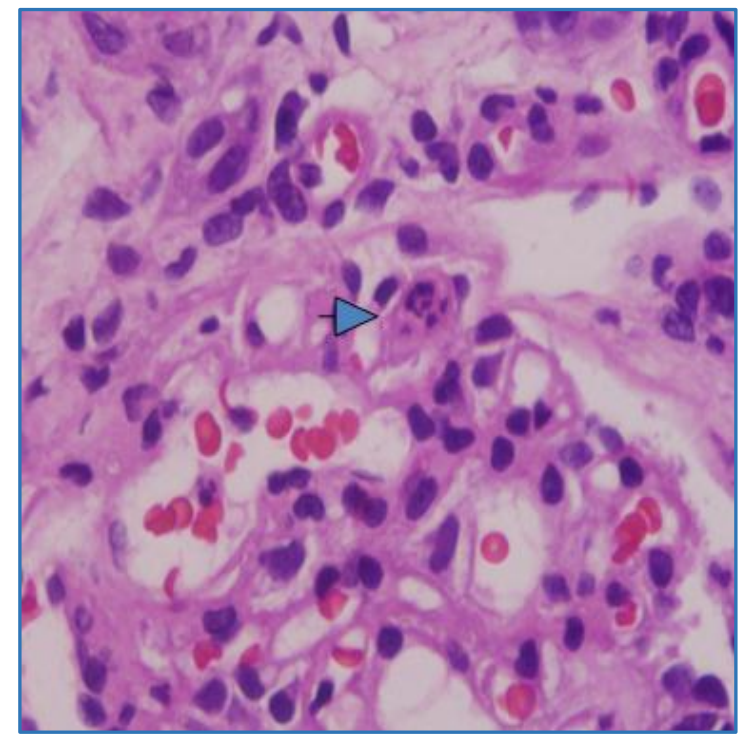

Figure 2. Neutrophilic Glomerulitis

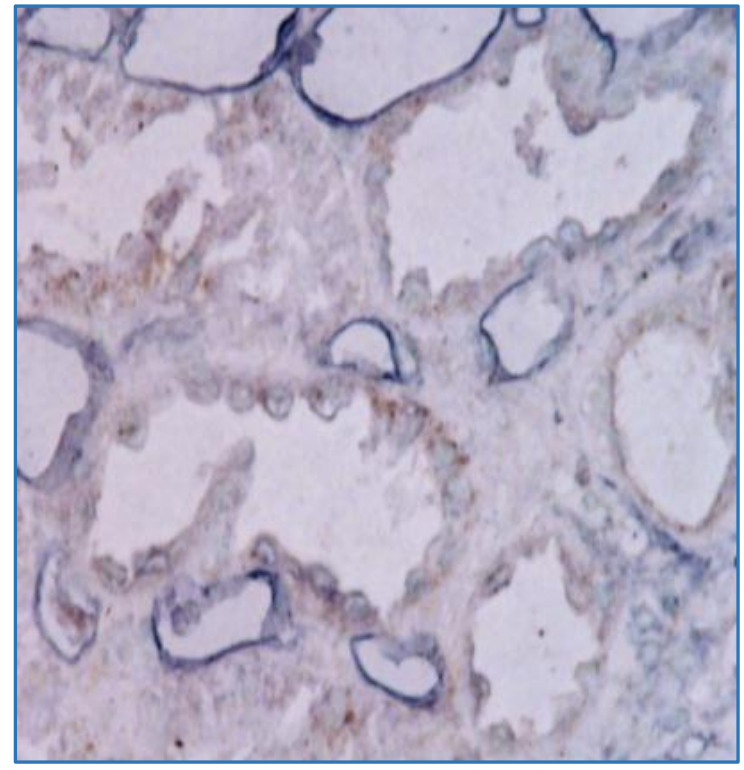

Figure 3. C4d Negative PTCS

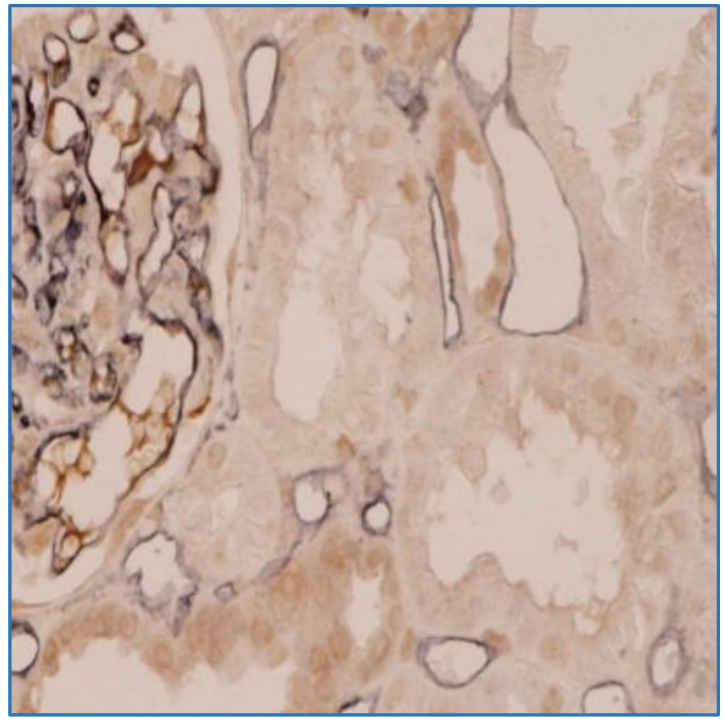

Figure 4. Glomeruli C4d Positive

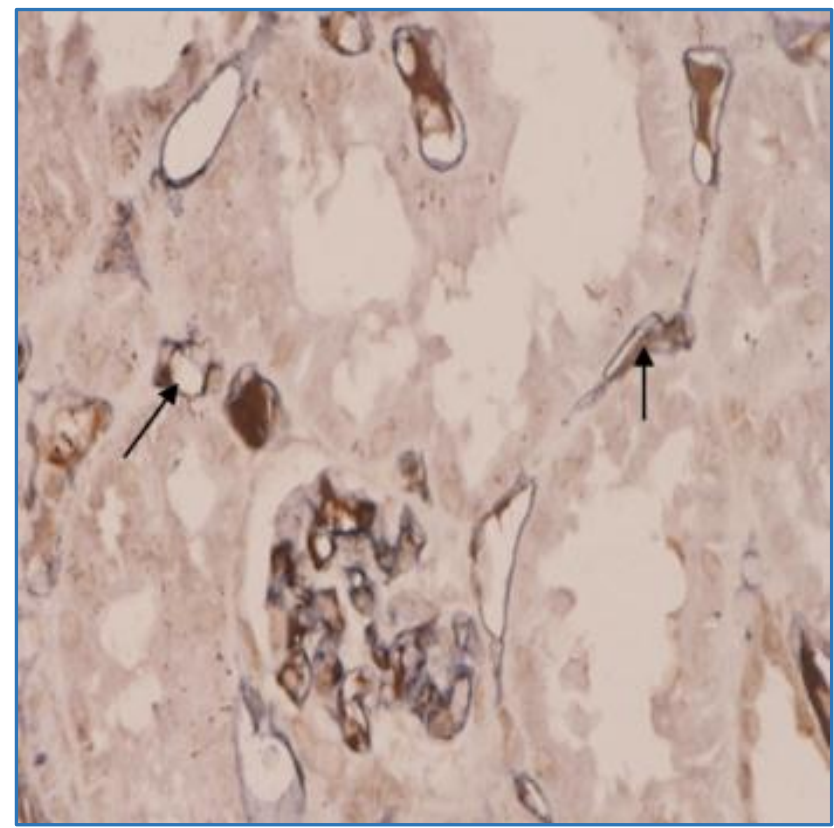

Figure 5. Focal C4d Positive

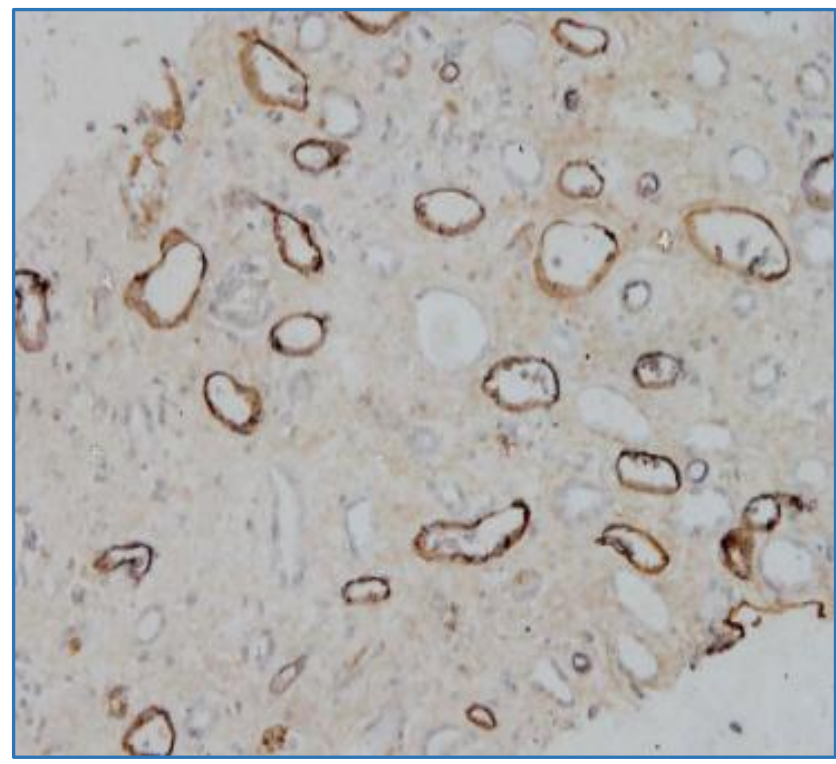

Figure 6. Diffuse C4d Positive

\section{DISCUSSION}

Statistically significant association $(\mathrm{p}<0.05)$ was found between the grade of rejection and the C4d positivity. Higher the grade of acute cellular rejection, more the chances of getting C4d deposition in peritubular capillaries.

\begin{tabular}{|c|c|c|}
\hline $\begin{array}{c}\text { Banff } \\
\text { Grade }\end{array}$ & $\begin{array}{c}\text { C4d Positive } \\
(\mathbf{n = 1 2 )} \\
(\mathbf{\%})(\mathbf{p}=\mathbf{0 . 1 5 4})\end{array}$ & $\begin{array}{c}\text { C4d Negative } \\
\mathbf{( n = 3 8 )} \\
(\mathbf{\%})(\mathbf{p}=\mathbf{0 . 1 5 4})\end{array}$ \\
\hline ACR+CAN & $7(58)$ & $12(32)$ \\
\hline Pure ACR & $3(26)$ & $5(13)$ \\
\hline Pure CAN & $1(8)$ & $10(26)$ \\
\hline $\begin{array}{c}\text { No } \\
\text { Rejection }\end{array}$ & $1(8)$ & $11(29)$ \\
\hline \multicolumn{3}{|c|}{ Table 1. Banff Grade } \\
\hline
\end{tabular}




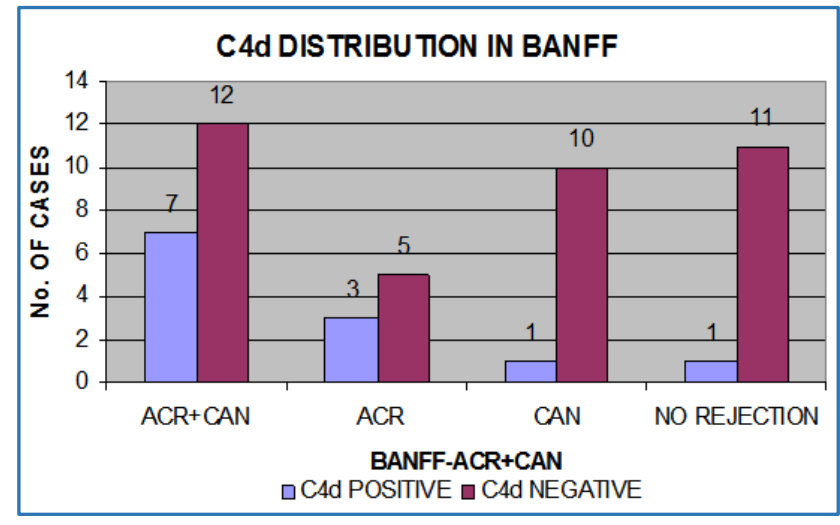

Association of C4d deposition on various grades of rejection was not statistically significant. Among the $\mathrm{C} 4 \mathrm{~d}$ positive cases $(n=12), 4$ cases of combined (ACR+CAN) rejection (4/7) were completely not responded to the treatment. One case of non-rejection biopsy also did not respond to the routine immunosuppressive treatment; 3 cases of pure acute cellular rejection responded completely to the treatment (Table 2).

\begin{tabular}{|c|c|c|}
\hline $\begin{array}{c}\text { C4d } \\
\text { Positive } \\
\text { Cases } \\
\end{array}$ & $\begin{array}{c}\begin{array}{c}\text { Responders } \\
(n=40)\end{array} \\
(\%)(p=0.231)\end{array}$ & $\begin{array}{c}\text { Non-Responders } \\
(n=10)(\%) \\
(p=0.231)\end{array}$ \\
\hline $\begin{array}{c}\text { No } \\
\text { Rejection }\end{array}$ & $0(0)$ & $1(10)$ \\
\hline ACR & $3(8)$ & $0(0)$ \\
\hline CAN & $1(3)$ & $0(0)$ \\
\hline ACR+CAN & $3(8)$ & $4(40)$ \\
\hline \multicolumn{3}{|c|}{ Table 2. C4d Positive Cases } \\
\hline
\end{tabular}

In this study 8 cases showed focal C4d positive capillaries (67 percent); 4 cases showed diffuse C4d positive capillaries (33 percent). On comparing the degree of C4d deposition with the response to anti-rejection treatment, 6 cases ( 75 percent) of focal C4d positive capillaries showed good response to treatment; 3 cases (75 percent) of diffusely C4d positive capillaries showed resistance to anti-rejection treatment. But its association was not statistically significant.

Mixed humoral and cellular rejection may be present in as much as 50 to 60 percent of all acute rejection episodes. ${ }^{17}$ Studies $^{7}$ were observed that the presence of humoral in addition to cellular rejection is a more severe antigraft reaction than cellular rejection alone and emphasised the importance of recognition of this variant of acute rejection because clinical intervention by treatment of humoral rejection has the potential to alleviate graft injury. Treatment strategies ${ }^{18}$ for acute antibody-mediated rejection are based on the quick reduction of antibody titres with plasmapheresis plus immunosuppression with drugs such as tacrolimus and mycophenolate mofetil.

Histologically, normal protocol biopsies may show C4d deposition.19 Further followup and correlations with circulating antibody will be needed to interpret the clinical significance of incidental C4d deposition. ${ }^{19}$

Studies ${ }^{20,21}$ have shown the direct comparison of the sensitivity of Immunofluorescence (IF) to C4d in frozen sections with Immunoperoxidase stains using $\mathrm{C} 4 \mathrm{~d}$ in paraffin embedded tissue has been reported. False negative rate of IHC (C4d alone) is roughly about 10 to 20 percent and that of IF is 5 to 10 percent. ${ }^{21,22}$

\section{CONCLUSION}

Recognition of humoral rejection may be problematic in biopsies with extensive inflammation and all the posttransplant biopsies should be screened for humoral component irrespective of their rejection status. Dual Immunostaining with CD34 is very useful to define the C4d positivity and also to differentiate between the focal or diffuse positivity. No study has used simultaneous endothelial cell marker (CD34/Factor VIII) for identification of PTC. Dual IHC will also reduce the chances of false negative detection of humoral components in allograft biopsy.

\section{REFERENCES}

1. Bourne JA. Handbook of immunoperoxidase staining methods. Immunohistochemistry Laboratory, DAKO corporation, 1983.

2. Racusen LC, Colvin RB, Solez K, et al. Antibody mediated rejection criteria an addition to the banff 97 classification of renal allograft rejection. Am J Transplant 2003;3(6):708-14.

3. Hume DM, Merill JP, Miller BF, et al. Experiences with renal homotransplantations in the human: report of nine cases. Journal of Clinical Investigations 1955;34(2):32782.

4. Evans RW, Kitzmann DJ. An economic analysis of kidney transplantation. Surg Clin North Am 1998;78(1):149-74.

5. Cecka JM. The OPTN/UNOS renal transplant registry. In: Clinical transplants 2003. Los angles: UCLA Immunogenetics Center, 2004:1.

6. Racusen LR, Solez K, Colvin RB, et al. The banff 97 working classification of renal allograft pathology. Kidney International 1999;55(2):713-23.

7. Mauiyyedi S, Crespo M, Collins AB, et al. Acute humoral rejection in kidney transplantation. II. morphology, immunopathology and pathologic classification. J Am Soc Nephrol 2002;13(3):779-87.

8. Crespo M, Pascual M, Tolkoff-Rubin N, et al. Acute humoral rejection in renal allograft recipients. I. incidence, serology and clinical characteristics. Transplantation 2001;71(5):652-8.

9. Feucht HE, Felber E, Gokel MJ, et al. Vascular deposition of complement split products in kidney allografts with cell mediated rejection. Clin Exp Immunol 1991;86(3):46470.

10. Feucht HE, Schneeberger H, Hillebrand G, et al. Capillary deposition of $\mathrm{C} 4 \mathrm{~d}$ complement fragment and early renal graft loss. Kidney Int 1993;43(6):1333-8.

11. Ichida S, Yuzawa $\mathrm{Y}$, Okada $\mathrm{H}$, et al. Localization of the complement regulatory proteins in the normal human kidney. Kidney Int 1994;46(1):89-96.

12. Cosio FG, Sedmak DD, Mahan JD, et al. Localization of decay accelerating factor in normal and diseases kidneys. Kidney Int 1989;36(1):100-7.

13. Poduval RD, Kadambi PV, Josephon MA, et al. Implications of immunohistochemical detection of C4d along peritubular capillaries in late acute renal allograft rejection. Transplantation 2005;79(2):228-35.

14. Solez K, Axelsen RA, Benldiktsson H, et al. International standardization of criteria for the histological diagnosis of an renal allograft rejection: the banff working classification of kidney transplant pathology. Kidney Int 1993;44(2):411-22. 
15. Nickeleit V, Zeiler M, Gudat F, et al. Detection of the complement degradation product $\mathrm{C} 4 \mathrm{~d}$ in renal allografts: diagnostic and therapeutic implications. J Am Soc Nephrol 2002;13(1):242-51.

16. Racusen L, Colvin RB, Solez K, et al. Standardization of scoring of $\mathrm{C} 4 \mathrm{~d}$ staining in renal allograft biopsies: consensus from the seventh banff conference on allograft pathology. J Am Soc Nephrol 2003;14:146A-7A.

17. Trypkov K, Campbell P, Pazderka F, et al. Pathologic features of acute renal allograft rejection associated with donor-specific antibody: analysis using the banff grading schema. Transplantation 1996;61(11):1586-92.

18. Terasaki PI, Ozawa M. Predicting kidney graft failure by HLA antibodies: a prospective trial. Am J Transplant 2004;4(3):438-43.
19. Mengel M, Bogers J, Bosmans JL, et al. Incidence of C4d stain in protocol biopsies from renal allografts: results from a multicenter trial. Am J Transplant 2005;5(5):10506.

20. Regele H, Exner M, Watschinger B, et al. Endothelial C4d deposition is associated with inferior kidney allograft outcome independently of cellular rejection. Nephrol Dial Transplant 2001;16(10):2058-66.

21. Troxell ML, Weintraub LA, Higgin JP, et al. Comparison of C4d immunostaining methods in renal allograft biopsies. CJASN 2006;1(3):583-91.

22. Nadasdy GM, Bott C, Cowden D, et al. Comparative study for the detection of peritunular capillary C4d deposition in human renal allografts using different methodologies. Hum Pathol 2005;36(11):1178-85. 